\title{
Dry Eye in Video Display Terminal Users
}

\author{
Expert Interview with Gemma Caterina Maria Rossi
}

Clinica Oculistica, Fondazione IRCCS Policlinico San Matteo, Pavia, Italy

DOl: https://doi.org/10.17925/EOR.2019.13.2.67

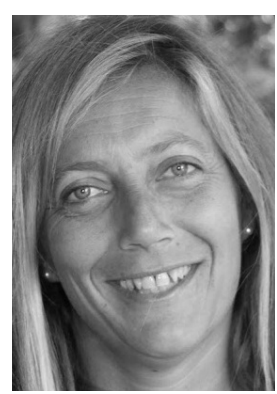

Gemma Caterina Maria Rossi

Dr Rossi graduated from the Medicine and Surgery Faculty of the University of Pavia in 1995 and the School for Ophthalmology Specialization at the University of Pavia in 2000 with full marks and honours. In 2001 she worked at the Eye Unit of the Azienda Ospedaliera Bolognini, Bg, as ophthalmic medical consultant and was responsible for the Glaucoma Centre of the Unit. Since 2008 she has worked at the Eye Clinic of the University of Pavia, Fondazione IRCCS Policlinico San Matteo, as ophthalmic medical consultant, residing at the Glaucoma Center of the Clinic and since 2018 she also works at the Retina Centre of the Clinic. She is a contract professor at the School for Ophthalmology Specialization at the University of Pavia. Dr Rossi has been involved as principal investigator in several national and international clinical trials on glaucoma therapy and ocular surface diseases. Since September 1998 she has been involved in the evaluation of the quality of life in glaucoma patients, more recently also in patients affected by ocular graft-vs-host disease and video display terminal workers. She won the MSD International 2006 award.

\section{Keywords}

Dry eye, video display terminal, VDT, occupational health, ocular surface, quality of life, prevalence

Disclosure: Gemma Caterina Maria Rossi is a consultant for Novartis and Santen.

Review Process: This is an expert interview and as such has not undergone the journal's standard peer review process.

Compliance with Ethics: This article is an expert interview and does not report on new clinical data, or any studies with human or animal subjects performed by any of the authors.

Authorship: The named author meets the International Committee of Medical Journal Editors (ICMJE) criteria for authorship of this manuscript, takes responsibility for the integrity of the work as a whole, and has given final approval for the version to be published.

Received: 8 November 2019

Published Online: 20 December 2019

Citation: European Ophthalmic Review. 2019;13(2):67-8

Corresponding Author: Gemma Caterina Maria Rossi, IRCCS Policlinico San Matteo Foundation, P.le Golgi 19, Pavia 27100, Italy. E: gemma.rossi.md@gmail.com

Support: No funding was received in

the publication of this article.
$\mathrm{D}$ ry eye is a common ocular condition that results in eye pain and discomfort, visual disturbance and substantially affects quality of life. ${ }^{1}$ The incidence of dry eye is markedly increasing due to the expansion in the global internet network. ${ }^{2}$ The use of video display terminals (VDT) and other devices, including desktops, laptops, tablets, and smartphones is now part of daily life, worldwide. The incidence of dry eye is particularly high in workers that work with VDT. ${ }^{3}$ A recent study has estimated the prevalence of dry eye among VDT users and assessed risk factors for dry eye in this population. ${ }^{4}$ In an expert interview, lead author Gemma Rossi discusses this study and the significance of its findings.

\section{Q. What was previously known about the prevalence of dry eye in VDT workers?}

So far, data on the prevalence of dry eye in VDT workers have referred to two large studies on the Asian population: the Osaka study ${ }^{5}$ and the Moriguchi study. ${ }^{6}$ These studies found a prevalence of dry eye that ranged from $4-87 \%$ of workers. The heterogeneous prevalence of the condition depended on diagnostic criteria of dry eye.

\section{Q. Could you tell us a little about your study's objectives and design?}

The main objective of our study was to estimate the prevalence of dry eye among Caucasian VDT users and a control group, applying the same diagnostic criteria, which has not been attempted before. ${ }^{4}$ Secondly, we investigated the risk factors for developing dry eye in this population, considering age, gender, number of years spent working at a VDT, number of effective hours a day at a VDT, and number and duration of breaks a day taken from VDT use.

\section{Q. What were the main findings of this study?}

Our study included 194 Caucasian subjects, and found that $24.2 \%$ of VDT workers presented a definite dry eye versus $2.8 \%$ of controls; moreover, subjects with dry eye had a lower quality of life in comparison to those without dry eye, when investigated with a specific questionnaire. The data analysis revealed that the age and the time at a VDT are associated with dry eye: with a 1.05-fold increase in risk per year, and 1.57-fold increase in risk per each 2-hour increase at a VDT, respectively.

\section{Q. What are the wider implications of these findings?}

Increased age and the number of hours spent on computers are the main risk factors for developing dry eye; this suggests the need to think and propose preventive measures to avoid the onset or the worsening of dry eye. Our study has revealed that symptoms are an important component of dry; therefore, an accurate anamnesis on symptoms should be always done. Moreover, we found that many subjects presented a reduced tear break-up time not justified by gender and age, but 
related to environmental parameters that may represent a risk factor for visual fatigue.

\section{Q. How can dry eye be prevented in people using computers?}

Workers who spend more time at a VDT need not only ergonomics and environmental improvements, but also specific ophthalmic evaluations and treatments. The evaluation of tear film status (breakup time, corneal staining, meibomian gland disfunction) and symptom investigation should be regularly performed on all VDT workers. The use of lacrimal substitutes can improve ocular discomfort, and improve both quality of life and productivity. The ophthalmologist's task is to prescribe artificial tears to prevent dry eye disorders instead of trying to remedy them. $\square$
1. The definition and classification of dry eye disease: report of the Definition and Classification Subcommittee of the International Dry Eye WorkShop (2007). Ocul Surf. 2007:5:75-92

2. Wolkoff P. Ocular discomfort by environmental and personal risk factors altering the precorneal tear film. Toxicol Lett. 2010;199:203-12.
3. Nakamura S. Approach to dry eye in video display terminal workers (basic science). Invest Ophthalmol Vis Sci. 2018:59:DES130-DES137.

4. Rossi GCM, Scudeller L, Bettio F, et al. Prevalence of dry eye in video display terminal users: a cross-sectional Caucasian study in Italy. Int Ophthalmol. 2019;39:1315-22
5. Uchino M, Uchino Y, Dogru M, et al. Dry eye disease and work productivity loss in visual display users: the Osaka study. Am J Ophthalmol. 2014;157:294-300.

6. Kawashima M, Yamatsuji M, Yokoi N, et al. Screening of dry eye disease in visual display terminal workers during occupational health examinations: The Moriguchi study. I Occup Health. 2015;57:253-8. 